\title{
XTRPC1-dependent chemotropic guidance of neuronal growth cones
}

\author{
Sangwoo Shim ${ }^{1,2}$, Eyleen L Goh ${ }^{1,2}$, Shaoyu Ge ${ }^{1,2}$, Kurt Sailor $^{1,2}$, Joseph P Yuan ${ }^{3}, \mathbf{H}$ \\ Llewelyn Roderick ${ }^{4}$, Martin D Bootman ${ }^{4}$, Paul F Worley ${ }^{3}$, Hongjun Song ${ }^{1,2,3}$, and Guo-li \\ Ming ${ }^{1,2,3}$ \\ ${ }^{1}$ Institute for Cell Engineering, Johns Hopkins University School of Medicine, 733 N. Broadway, \\ Baltimore, Maryland 21205, USA \\ ${ }^{2}$ Department of Neurology, Johns Hopkins University School of Medicine, 733 N. Broadway, \\ Baltimore, Maryland 21205, USA \\ ${ }^{3}$ Department of Neuroscience, Johns Hopkins University School of Medicine, 725 N. Wolfe Street, \\ Baltimore, Maryland 21205, USA \\ ${ }^{4}$ Laboratory of Molecular Signalling, The Babraham Institute, Babraham, Cambridge CB2 4AT, \\ UK
}

\begin{abstract}
Calcium arising through release from intracellular stores and from influx across the plasma membrane is essential for signalling by specific guidance cues and by factors that inhibit axon regeneration. The mediators of calcium influx in these cases are largely unknown. Transient receptor potential channels (TRPCs) belong to a superfamily of $\mathrm{Ca}^{2+}$-permeable, receptoroperated channels that have important roles in sensing and responding to changes in the local environment. Here we report that XTRPC1, a Xenopus homolog of mammalian TRPC1, is required for proper growth cone turning responses of Xenopus spinal neurons to microscopic gradients of netrin-1, brain-derived neurotrophic factor and myelin-associated glycoprotein, but not to semaphorin 3A. Furthermore, XTRPC1 is required for midline guidance of axons of commissural interneurons in the developing Xenopus spinal cord. Thus, members of the TRPC family may serve as a key mediator for the $\mathrm{Ca}^{2+}$ influx that regulates axon guidance during development and inhibits axon regeneration in adulthood.
\end{abstract}

\begin{abstract}
Axons are guided to their targets in the developing nervous system by diffusible and bound cues that either attract or repel the growing tip of an axon, the growth cone ${ }^{1-4}$. Once neuronal circuits are formed, the ability of axons to regenerate after injury or disease in the mature mammalian CNS is extremely limited, largely because of the presence of inhibitory factors ${ }^{5-10}$. During the last decade, an array of evolutionarily conserved guidance cues and
\end{abstract}


their receptors have been identified and have been shown to be involved in axon guidance during development and in axon regeneration in the adult central nervous system ${ }^{1-3,6}$. How specific guidance signals are transduced from receptor activation to rearrangement of cytoskeleton within the neuronal growth cone is just beginning to be elucidated ${ }^{1-4,11,12}$. One of the initial signal transduction mechanisms that triggers the growth cone responses to a large number of guidance cues and inhibitory factors associated with myelin is an increase in cytosolic $\mathrm{Ca}^{2+}$, arising from both intracellular store release and influx across the plasma membrane ${ }^{5,7,13-17}$. Notably, different patterns of $\mathrm{Ca}^{2+}$ elevation trigger differential attractive and repulsive turning responses of the growth cone ${ }^{13,18}$. While previous studies have demonstrated the essential role of $\mathrm{Ca}^{2+}$ signalling in axon guidance and regeneration both in vitro and in vivo $5,7,11-13,15-24$, it is unknown how initial $\mathrm{Ca}^{2+}$ mobilization from intracellular stores is coupled to sustained $\mathrm{Ca}^{2+}$ influx from the extracellular environment to regulate growth cone guidance ${ }^{11,12}$.

Members of the TRPC family (TRPC1-TRPC7 in mammals) are non-selective cation channels; thus, they are good candidates to mediate sustained $\mathrm{Ca}^{2+}$ influx ${ }^{25,26}$. For example, TRPC 3 and TRPC1 mediate $\mathrm{Ca}^{2+}$ influx induced by brain-derived neurotrophic factor $(\mathrm{BDNF})^{27}$ and by activation of metabotropic glutamate receptor mGluR1 (ref. 28), respectively. Notably, TRPC5 regulates growth cone morphology and neurite extension in cultured hippocampal neurons ${ }^{29,30}$. XTRPC1, a homolog of mammalian TRPC1, is the only TRPC family member cloned so far from Xenopus ${ }^{31,32}$. In this study, with morpholinomediated gene knockdown, expression of dominant-negative channels and pharmacological inhibitors, we examined the role of XTRPC1 in Xenopus spinal neuron growth cone turning responses to gradients of specific guidance cues and inhibitory factors for axon regeneration, including netrin-1, BDNF, myelin-associated glycoprotein (MAG) and semaphorin 3A (Sema3A). Furthermore, we examined the role of XTRPC1 in the CNS midline axon guidance of commissural interneurons in developing Xenopus spinal cord in vivo, a classic example of long-range axon guidance by netrin-1 (refs. 33-38). Our in vitro and in vivo studies provide strong evidence that XTRPC1 is required by a select group of guidance cues for signalling. Two recent independent in vitro studies have also demonstrated roles for TRPC1 and TRPC 3 in attractive growth cone responses to netrin- 1 and BDNF 39,40 . Together with recent findings on the roles of TRPs in various biological behaviors ${ }^{25,26,41}$, these studies support the view that TRP superfamily members function as general cellular sensors for extracellular environments, including sensing guidance cues during neuronal pathfinding.

\section{RESULTS}

\section{Expression of XTRPC1 at growth cones of spinal neurons}

We first examined the expression of XTRPC 1 in Xenopus spinal neurons with previously characterized antibodies ${ }^{31}$. We found that XTRPC1 was expressed in the majority of Xenopus spinal neurons in the culture (Fig. 1a). Notably, we observed enriched staining of XTRPC1 at growth cones of some neurons (Fig. 1a). When injected into the early Xenopus embryos, a morpholino specific for XTRPC1, but not a control morpholino, substantially reduced the expression level of XTRPC1 in cultured spinal neurons derived from injected 
embryos, as shown by immunocytochemistry (Fig. 1b,c,e), and in whole embryos, as shown by western blot (Fig. 1d,e; see Methods).

\section{XTRPC1 in netrin-1-induced growth cone attraction}

To examine the functional role of XTRPC1 in axon guidance, we used a well-established in vitro growth cone turning assay ${ }^{13-17}$. In a microscopic gradient of netrin-1 $(5 \mu \mathrm{g} / \mathrm{ml}$ in the pipette), Xenopus spinal neurons demonstrated chemoattractive turning responses under normal conditions (Fig. 1f). When spinal neurons derived from embryos injected with the XTRPC1 morpholino were exposed to the same netrin-1 gradient, the attraction was completely abolished (Figs. $1 \mathrm{~g}$ and 2). Instead, we observed significant repulsive turning responses (Figs. 1g and 2b). However, a control morpholino had no effect on netrin-1induced attraction (Fig. 2c). As the morpholino treatment did not completely eliminate the expression of XTRPC1 (Fig. 1e), these results suggest that the expression level of XTRPC1 is critical for the proper growth cone responses to a netrin-1 gradient. XTRPC1 shows a high degree of homology to the mammalian TRPC1, especially at the proposed pore region (Fig. 2a). We found that netrin-1-induced attraction was partially rescued when mRNAs encoding wild-type mammalian TRPC1 were co-injected with the XTRPC1 morpholino (Fig. 2c), further demonstrating the role of TRPC1 in growth cone turning to netrin-1. TRPCs with specific mutations at the pore region have been previously shown to block endogenous channel opening and act in a dominant negative fashion 28,30 . We found that expression of a mutant form of mammalian TRPC1, TRPC1(F562A) ${ }^{28}$, converted netrin-1-induced attraction into repulsion (Figs. $1 \mathrm{~h}$ and 2c), very similar to the effect of XTRPC1 morpholino treatment. The neurite extension rate in a netrin-1 gradient was not statistically different under these conditions (Supplementary Fig. 1). When neurons were cultured in the absence of guidance cues, neurite length was also not significantly different under different conditions (Supplementary Fig. 1). In addition to genetic manipulation, SKF96365 (2 $\mu \mathrm{M})$ and $\mathrm{LaCl}_{3}(10 \mu \mathrm{M})$, two commonly used inhibitors for TRPCs and other $\mathrm{Ca}^{2+}$ channels, also abolished attractive growth cone responses to a gradient of netrin-1 (Fig. 2c). Taken together, these results demonstrate that XTRPC1 is required for the attractive responses by Xenopus spinal neuron growth cones to a netrin-1 gradient in vitro. Previous studies have shown that an elevation of cytoplasmic $\mathrm{Ca}^{2+}$ owing to intracellular store release and influx from the extracellular environment are both required for proper growth cone turning responses to netrin-1 (refs. 13,15). Removal of extracellular $\mathrm{Ca}^{2+}$ converts growth cone attraction induced by focal laser-induced photolysis of caged $\mathrm{Ca}^{2+}$ to repulsion ${ }^{18}$. Thus, our results are consistent with the notion that XTRPC1 is an essential component of the cellular machinery mediating $\mathrm{Ca}^{2+}$ influx in response to netrin-1.

\section{XTRPC1 in formation of commissural interneuron axon tracts}

We next examined whether XTRPC1 is also required for CNS midline guidance of commissural interneuron axons in vivo, a classic example of netrin-1-dependent long-range growth cone guidance $1,3,37,38$. Disruption of netrin-1-dependent signalling has been shown to result in significant defects in the formation of the contralateral longitudinal commissural interneuron axon tracts in developing spinal cords in multiple model systems ${ }^{34,36,37,42,43}$. To directly compare the axon tracts of neurons derived from the injected and uninjected blastomere in the same embryo, we injected the XTRPC1 morpholino into one of the two 
blastomeres at the two-cell stage, together with a lineage tracer (Fig. 3a; see Methods). In the developing Xenopus spinal cord, commissural interneurons send axons ventrally to cross the midline, where they usually branch to ascend and descend in the marginal zone (Fig. 3b; ref. 44). We used the monoclonal antibody 3A10 to identify axons of commissural interneurons 37,45 . Whole-mount staining of stage 28-30 Xenopus embryos with 3A10 reveals two longitudinal axon tracts of commissural interneurons on each side of the midline as well as crossing axons within the developing spinal cord (Fig. 3c). Since these commissural interneurons have only contralateral projections ${ }^{44}$, the fibers on the contralateral side of the midline reflect those that had crossed the midline (Fig. 3b). We found that contralateral longitudinal axons of commissural interneurons derived from the XTRPC1 morpholino-injected blastomere were significantly less thick than those from the uninjected blastomere in the same embryo in a ventral view (Fig. 3d,f and Supplementary Video 1). We also observed this reduction in sagittal views of 3A10-positive fibers from the XTRPC1 morpholino-injected side, compared with controls, in the same segment of the spinal cord (Supplementary Fig. 2). Injection of a control morpholino, however, had no effect (Fig. 3e,f and Supplementary Video 2). In addition, injection of mRNA encoding wild-type mammalian TRPC1 together with the XTRPC1 morpholino largely rescued the formation of contralateral longitudinal commissural interneuron axons from the injected side (Fig. 3f). Thus, XTRPC1 is required for proper formation of contralateral longitudinal commissural axon tracts in vivo. To further examine whether observed defects on commissural axon tracts is due to a reduction of the number of commissural interneurons, we examined sagittal views of 3A10-positive neurons at both sides of the spinal cord of embryos injected unilaterally at the two-cell stage (Supplementary Fig. 2). We found that the density of commissural interneurons labelled with 3A10 in the injected embryos was very similar to that previously reported at same developmental stages using different markers for commissural interneurons ${ }^{46}$. More importantly, we did not observe any significant difference in the number of 3A10-positive neurons between the uninjected side and the side injected with the XTRPC1 morpholino within the same segment of spinal cord (Supplementary Fig. 2).

\section{XTRPC1 in attracting commissural axons to the CNS midline}

To directly examine whether interfering with XTRPC1 functions leads to failure to attract commissural axons to the CNS midline in vivo, we examined spinal cords from a sagittal view of stage 23-25 embryos when commissural interneuron axons just crossed the midline and major tracts started to form (Fig. 4a). We found that almost all 3A10-positive commissural axons derived from uninjected blastomeres or from blastomeres injected with the control morpholino crossed the midline at this stage (Fig. 4b,d,f). In contrast, a significant percentage of 3A10-positive commisural axons derived from blastomeres injected with the XTRPC1 morpholino failed to cross the midline $(35 \pm 3.7 \%, n=23$ embryos; $P<0.01$; Fig. 4f). Out of 459 commissural interneurons derived from 23 embryos unilaterally injected with the XTRPC1 morpholino (Fig. 4c,d), some of them branched prematurely $(4.8 \pm 1.2 \%)$ or joined the ipsilateral axonal tract $(13.9 \pm 2.2 \%)$, and others seemed to stall or even turn back from the midline $(18.7 \pm 2.8 \%)$. We did not observe a significant difference between the injected and uninjected side in the number of $3 \mathrm{~A} 10$ positive neurons within the same segment of spinal cord (Fig. 4e) or in the intensity of 3A10 
staining of labelled axons (Fig. 4). Taken together, these results suggest that XTRPC1 is essential for midline axon guidance of commissural interneurons, a classic example of netrin-1-dependent axon guidance ${ }^{37,38}$.

\section{XTRPC1 as a common mediator for a group of guidance cues}

To further examine whether XTRPC1 serves as a common mediator for multiple guidance factors, we examined the turning responses of Xenopus spinal neurons to microscopic gradients of MAG and Sema3A, both of which have been implicated in inhibition of axon regeneration in the adult $\mathrm{CNS}^{3,6,8,10}$. Xenopus spinal neurons demonstrated repulsive turning responses to a gradient of MAG $(150 \mu \mathrm{g} / \mathrm{ml} \text { in the pipette; Fig. } 5 \mathrm{a})^{14,16,47}$ and to a gradient of Sema3A (50 $\mu \mathrm{g} / \mathrm{ml}$ in the pipette; Fig. $5 \mathrm{~b})^{16,47}$. We found that interfering with XTRPC1 signalling, either by injection of XTRPC1 morpholino or by injection of mRNA encoding DN-TRPC1, completely abolished MAG-induced repulsion (Fig. 5a,c). In contrast, Sema3A-induced repulsion was not significantly affected $(P>0.05)$ in neurons injected with the XTRPC1 morpholino (Fig. 5b,c). We have previously shown that growth cone responses of Xenopus spinal neurons to a gradient of netrin-1, MAG or BDNF, but not to a gradient of Sema3A, are abolished when extracellular $\mathrm{Ca}^{2+}$ are reduced from $1 \mathrm{mM}$ to $1 \mu \mathrm{M}$, indicating a differential requirement for $\mathrm{Ca}^{2+}$ influx in the signalling events triggered by these two groups of factors ${ }^{16}$. Furthermore, we found that attraction to a BDNF gradient $(50 \mu \mathrm{g} / \mathrm{ml}$ in the pipette) was also abolished in neurons derived from XTRPC1 morpholino-injected blastomeres (Fig. 5c). Thus, our results suggest that XTRPC1 may be a key mediator of $\mathrm{Ca}^{2+}$ influx for a select group of guidance cues, including factors that inhibit axon regeneration in the adult CNS.

\section{DISCUSSION}

Our study provides both in vivo and in vitro evidence that XTRPC1 is required for guiding neuronal growth cones in response to specific guidance cues. Early studies have shown that patterns of $\mathrm{Ca}^{2+}$ elevation, (for example, initial $\mathrm{Ca}^{2+}$ transients and basal levels of cytoplasmic $\mathrm{Ca}^{2+}$ ) regulate the directionality of growth cone turning ${ }^{13,18}$. TRPCs are nonselective cation channels, and they can contribute to the influx of $\mathrm{Ca}^{2+}$ upon stimulation by specific guidance cues at least in two ways (Supplementary Fig. 3). First, TRPCs are $\mathrm{Ca}^{2+}-$ permeable channels, thus allowing direct influx of $\mathrm{Ca}^{2+}$. Second, activation of TRPCs also leads to influx of $\mathrm{Na}^{+}$and subsequent membrane depolarization, which may activate voltage-gated $\mathrm{Ca}^{2+}$ channels and result in further $\mathrm{Ca}^{2+}$ influx ${ }^{13}$. Indeed, recent studies have shown that netrin-1 can induce TRPC-like currents in neuronal growth cones and subsequent membrane depolarization of these neurons in culture ${ }^{40}$. Interfering with XTRPC1 functions would substantially reduce the influx of extracellular $\mathrm{Ca}^{2+}$ after initial release of $\mathrm{Ca}^{2+}$ from intracellular stores, resulting in a smaller amplitude of $\mathrm{Ca}^{2+}$ transients and a lower level of global cytoplasmic $\mathrm{Ca}^{2+}$. Consequently, attractive growth cone responses to guidance cues such as netrin- 1 and BDNF are abolished ${ }^{40}$ or turned into repulsion (Figs. $2 \mathrm{c}$ and $5 \mathrm{c}$ ) depending on the level of XTRPC1 knockdown. Two recent independent studies have shown that TRPC 3 mediates $\mathrm{Ca}^{2+}$ influx in cerebellar granule neurons and is required for BDNF-induced attraction, and that XTRPC1 is required for intracellular $\mathrm{Ca}^{2+}$ elevation and attraction induced by netrin-1 (ref. 40). In the current study, we have used morpholino- 
mediated knockdown, expression of dominant-negative mutants and pharmacological inhibitors to interfere with XTRPC1 signalling. We show that XTRPC1 is not only involved in attractive signalling to netrin- 1 and BDNF but is also differentially required for signalling of two repulsive guidance cues (MAG and Sema3A; Fig. 5c). An emerging view from all of these in vitro findings is that different members of the TRPC family may be widely involved in different guidance behaviors of neuronal growth cones.

Netrin-1-dependent signalling is essential for midline axon guidance of commissural interneurons $34,36,37,42,43$. We provide the first in vivo evidence that XTRPC1 is involved in midline axon guidance of commissural interneurons in developing Xenopus spinal cord. In stage 28-30 embryos, we have observed a significant reduction in contralateral longitudinal commissural interneuron axon tracts derived from the side injected with the XTRPC1 morpholino than were derived from the uninjected side within the same spinal cords (Fig. 3 and Supplementary Fig. 2). This defect in the formation of contralateral commissural interneuron axon tracts is due to a failure of attracting some of the axons to the CNS midline. In the developing spinal cord of stage 23-25 embryos, we found that a significant number of commissural axons derived from blastomeres injected with the XTRPC1 morpholino did not reach the CNS midline (Fig. 4). We did not find any difference in the number of commissural interneurons with the XTRPC1 morpholino injection at the stages we examined (Fig. 3e; Supplementary Fig. 2e). Future mosaic analysis will be needed to prove that the observed defects are stringently cell-autonomous in vivo. As axons derived from the uninjected side can project across the midline normally and form the contralateral commissural axon tracts, a general defect of midline cells owing to the XTRPC1 morpholino treatment is unlikely. The pathfinding defects of commissural inter-neurons observed following XTRPC1 morpholino treatment, however, are likely to result from a disruption of growth cone responses to multiple guidance cues involved in midline guidance, including netrin-1. Our previous studies have shown that the turning responses to group I cues (including netrin-1, MAG and BDNF) are very sensitive to manipulation of extracellular $\mathrm{Ca}^{2+}$, whereas responses to group II cues (including Sema3A and NT-3) are not affected by the same manipulation $15,16,47,48$. Our current results suggest that XTRPC1 is involved in $\mathrm{Ca}^{2+}$ signalling for group I cues. Since multiple inhibitors associated with the CNS myelin are likely to belong to group I cues ${ }^{47}$, inhibition of the function of TRPCs may potentially promote axon regeneration in the injured adult CNS.

How XTRPC1 is activated by netrin-1, BDNF and MAG remains to be determined. Specific guidance cues can induce tyrosine phosphorylation of their receptors ${ }^{48,49}$, providing docking sites for proteins, such as phospholipase C- $\gamma$ (PLC- $\gamma$ ), that contain SH2 domains (Supplementary Fig. 3). Together with the requirement of PLC- $\gamma$ in growth cone turning 39,48 and activation of TRPCs by PLC ${ }^{25,26}$, our results suggest a model in which guidance cues or inhibitors for axon regeneration activate PLC, which in turn leads to release of intracellular stores of $\mathrm{Ca}^{2+}$ through the $\mathrm{IP}_{3}$ receptor and an influx of extracellular $\mathrm{Ca}^{2+}$ through TRPCs (Supplementary Fig. 3). Thus, members of the TRP superfamily may serve as a key component of the cellular machinery in sensing and responding to changes in the local environment in general, including pathfinding of neuronal growth cones in response to specific guidance cues. 


\section{METHODS}

\section{Xenopus embryo injection, cell culture, immunocytochemistry and biochemistry}

Blastomere injection of morpholino oligonucleotides and mRNAs encoding mammalian TRPC1 or its mutant forms into early stages of Xenopus embryos were performed as previously described ${ }^{48,49}$. Specifically, fertilized embryos were injected with a mixture of the morpholino ( 2 or 10 ng/embryo) or mRNA (2-3 ng/embryo), and a lineage tracer (fixable FITC-dextran or rhodamine-dextran, $15 \mu \mathrm{g} / \mu \mathrm{l}$ ), at the one- or two-cell stage. A morpholino oligo specific for XTRPC1 was designed with the following sequence: $5^{\prime}$ GAGCAGCCATGATGACAGAACTCCC- $3^{\prime}$. A control morpholino was used with the following sequence: $5^{\prime}$-CCTCTTACCTCAGTTACAATTTATA- $3^{\prime}$. Injected embryos at stage 22 were used for spinal neuron cultures as previously described ${ }^{48,49}$. For immunocytochemistry, cultured cells derived from embryos unilaterally injected at the twocell stage were processed as previously described ${ }^{48,49}$. Polyclonal antibodies against two different regions of XTRPC 1 have been previously characterized ${ }^{31}$ and were used at a dilution of 1:100 for immunocytochemistry of cell cultures and at a dilution of 1:1,000 for western blot. Similar results were obtained with both antibodies. Secondary antibodies were used at a dilution of 1:250. A majority of neurons (80-90\%) were stained for XTRPC1 in culture. Images of cultured neurons were captured with a Zeiss 510 confocal microscope using the same settings for different conditions, and fluorescence intensities in a defined box covering only the neuronal growth cone regions were quantified. Twenty stage 26 uninjected embryos or embryos injected exclusively at the one-cell stage, with either a control morpholino or the XTRPC1 morpholino, were used for membrane fractions and western blot analysis as described ${ }^{31,50}$. The total protein amount was measured, and an equivalent amount of proteins was loaded.

\section{Growth cone turning assay}

Microscopic gradients of guidance factors were generated using recombinant netrin-1 (5 $\mu \mathrm{g}$ / $\mathrm{ml})$, MAG (150 $\mu \mathrm{g} / \mathrm{ml})$, BDNF $(50 \mu \mathrm{g} / \mathrm{ml})$ or Sema3A (50 $\mu \mathrm{g} / \mathrm{ml})$, respectively, in the micropipette as previously described ${ }^{15,16,47,48}$. Neurons with the lineage-tracer were identified by fluorescence microscopy $14-20 \mathrm{~h}$ after plating and were used for the turning assay at $22-25{ }^{\circ} \mathrm{C}$ as described ${ }^{48,49}$. The turning angle was defined by the angle between the original direction of neurite extension and a straight line connecting the positions of the center of the growth cone at the onset and the end of the $30 \mathrm{~min}$ period. The rates of neurite extension were calculated based on the net neurite extension during the turning assay (Supplementary Fig. 1). Only growth cones of isolated neurons with a net neurite extension $>5 \mu \mathrm{m}$ over the 30-min period were included for turning analysis. Neurite lengths of neurons under different conditions cultured without addition of any guidance factors were also measured and quantified (Supplementary Fig. 1). Statistical significance $(P<0.05)$ was assessed using bootstrap test as indicated.

\section{Whole-mount immunocytochemistry and confocal imaging}

Normal embryos and embryos unilaterally injected at the two-cell stage were processed for whole-mount immunocytochemistry when they reached stage $23-25$ or 28-30 as previously described ${ }^{20}$. Monoclonal antibody 3A10 (Developmental Studies Hybridoma Bank at the 
University of Iowa), specific for commissural interneurons ${ }^{37,45}$, was used at a dilution of 1:100. Secondary antibodies were used at a dilution of 1:250. For ventral views of spinal cords, individual spinal cords were dissected out from Xenopus embryos at stage 28-30 and mounted on coverslips for imaging. Confocal images were taken with a Zeiss LSM 510 META system, and $z$-series reconstructions were processed with the Zeiss LSM image acquisition program. Three-dimensional (3D) projections were rotated with this software to ensure a direct ventral view of the spinal cord as shown in Fig. 3c-e and sample videos of rotating 3D images were also generated as shown in supplementary videos. Optical coronal section views of spinal cords were made from original $z$-stack confocal images (Fig. 3c-e) and were then used to measure mean area pixel intensities within a defined box containing either left or right longitudinal axon tracts for quantification (Fig. 3f). A minimum of three optical sections were made and measured for each sample, and a minimal of ten embryos were examined for each condition. For sagittal views of spinal cord, individual spinal cords were dissected out from stage 23-25 (Fig. 4) or 28-30 (Supplementary Fig. 2) embryos and were mounted within two layers of coverslips to allow imaging the same segment of the spinal cord from both sides for direct comparison. The density of 3A10-positive cells differs along the developing spinal cord. Thus, we directly compared the number of 3A10-positive cells in injected and uninjected sides within the same segment of developing spinal cord for each condition.

\section{Supplementary Material}

Refer to Web version on PubMed Central for supplementary material.

\section{Acknowledgments}

We would like to thank A. Kolodkin, C. Montell and T. Dawson for critical comments, N. Marsh-Armstrong, L.N. Borodinsky and N.C. Spitzer for their help during this study, and L. Liu for her technical support. This work was supported by National Institute of Neurological Disorders and Stroke, Charles E. Culpeper Scholarships in Medical Science, Whitehall Foundation, and Basal O'Connor Starter Scholar Research Award Program to G.L.M. S.S. is partially supported by a postdoctoral fellowship from the Korea Science and Engineering Foundation. H.L.R and M.D.B. would like to gratefully acknowledge support from the Biotechnology and Biological Sciences Research Council and Royal Society.

\section{References}

1. Tessier-Lavigne M, Goodman CS. The molecular biology of axon guidance. Science. 1996; 274:1123-1133. [PubMed: 8895455]

2. Yu TW, Bargmann CI. Dynamic regulation of axon guidance. Nat Neurosci. 2001; 4 (suppl):1169_ 1176. [PubMed: 11687826]

3. Huber AB, Kolodkin AL, Ginty DD, Cloutier JF. Signaling at the growth cone: ligand-receptor complexes and the control of axon growth and guidance. Annu Rev Neurosci. 2003; 26:509-563. [PubMed: 12677003]

4. Song H, Poo M. The cell biology of neuronal navigation. Nat Cell Biol. 2001; 3:E81-E88. [PubMed: 11231595]

5. Bandtlow CE, Schmidt MF, Hassinger TD, Schwab ME, Kater SB. Role of intracellular calcium in NI-35-evoked collapse of neuronal growth cones. Science. 1993; 259:80-83. [PubMed: 8418499]

6. He Z, Koprivica V. The Nogo signaling pathway for regeneration block. Annu Rev Neurosci. 2004; 27:341-368. [PubMed: 15217336]

7. Sivasankaran R, et al. PKC mediates inhibitory effects of myelin and chondroitin sulfate proteoglycans on axonal regeneration. Nat Neurosci. 2004; 7:261-268. [PubMed: 14770187] 
8. Fournier AE, Strittmatter SM. Repulsive factors and axon regeneration in the CNS. Curr Opin Neurobiol. 2001; 11:89-94. [PubMed: 11179877]

9. Schwab ME. Nogo and axon regeneration. Curr Opin Neurobiol. 2004; 14:118-124. [PubMed: 15018947]

10. Filbin MT. Myelin-associated inhibitors of axonal regeneration in the adult mammalian CNS. Nat Rev Neurosci. 2003; 4:703-713. [PubMed: 12951563]

11. Gomez TM, Spitzer NC. Regulation of growth cone behavior by calcium: new dynamics to earlier perspectives. J Neurobiol. 2000; 44:174-183. [PubMed: 10934320]

12. Henley J, Poo MM. Guiding neuronal growth cones using $\mathrm{Ca}^{2+}$ signals. Trends Cell Biol. 2004; 14:320-330. [PubMed: 15183189]

13. Hong K, Nishiyama M, Henley J, Tessier-Lavigne M, Poo M. Calcium signalling in the guidance of nerve growth by netrin-1. Nature. 2000; 403:93-98. [PubMed: 10638760]

14. Henley JR, Huang KH, Wang D, Poo MM. Calcium mediates bidirectional growth cone turning induced by myelin-associated glycoprotein. Neuron. 2004; 44:909-916. [PubMed: 15603734]

15. Ming GL, et al. cAMP-dependent growth cone guidance by netrin-1. Neuron. 1997; 19:1225-1235. [PubMed: 9427246]

16. Song $\mathrm{H}$, et al. Conversion of neuronal growth cone responses from repulsion to attraction by cyclic nucleotides. Science. 1998; 281:1515-1518. [PubMed: 9727979]

17. Song HJ, Ming GL, Poo MM. cAMP-induced switching in turning direction of nerve growth cones. Nature. 1997; 388:275-279. [PubMed: 9230436]

18. Zheng JQ. Turning of nerve growth cones induced by localized increases in intracellular calcium ions. Nature. 2000; 403:89-93. [PubMed: 10638759]

19. Berridge MJ. Neuronal calcium signaling. Neuron. 1998; 21:13-26. [PubMed: 9697848]

20. Gomez TM, Spitzer NC. In vivo regulation of axon extension and pathfinding by growth-cone calcium transients. Nature. 1999; 397:350-355. [PubMed: 9950427]

21. Wong ST, et al. A p75(NTR) and Nogo receptor complex mediates repulsive signaling by myelinassociated glycoprotein. Nat Neurosci. 2002; 5:1302-1308. [PubMed: 12426574]

22. Wen Z, Guirland C, Ming GL, Zheng JQA. CaMKII/calcineurin switch controls the direction of $\mathrm{Ca}(2+)-d e p e n d e n t$ growth cone guidance. Neuron. 2004; 43:835-846. [PubMed: 15363394]

23. Jin $\mathrm{M}$, et al. Ca2+-dependent regulation of rho GTPases triggers turning of nerve growth cones. J Neurosci. 2005; 25:2338-2347. [PubMed: 15745960]

24. Nishiyama M, et al. Cyclic AMP/GMP-dependent modulation of Ca2+ channels sets the polarity of nerve growth-cone turning. Nature. 2003; 423:990-995. [PubMed: 12827203]

25. Montell C, Birnbaumer L, Flockerzi V. The TRP channels, a remarkably functional family. Cell. 2002; 108:595-598. [PubMed: 11893331]

26. Moran MM, Xu H, Clapham DE. TRP ion channels in the nervous system. Curr Opin Neurobiol. 2004; 14:362-369. [PubMed: 15194117]

27. Li HS, Xu XZ, Montell C. Activation of a TRPC3-dependent cation current through the neurotrophin BDNF. Neuron. 1999; 24:261-273. [PubMed: 10677043]

28. Kim SJ, et al. Activation of the TRPC1 cation channel by metabotropic glutamate receptor mGluR1. Nature. 2003; 426:285-291. [PubMed: 14614461]

29. Bezzerides VJ, Ramsey IS, Kotecha S, Greka A, Clapham DE. Rapid vesicular translocation and insertion of TRP channels. Nat Cell Biol. 2004; 6:709-720. [PubMed: 15258588]

30. Greka A, Navarro B, Oancea E, Duggan A, Clapham DE. TRPC5 is a regulator of hippocampal neurite length and growth cone morphology. Nat Neurosci. 2003; 6:837-845. [PubMed: 12858178]

31. Bobanovic LK, et al. Molecular cloning and immunolocalization of a novel vertebrate trp homologue from Xenopus. Biochem J. 1999; 340:593-599. [PubMed: 10359642]

32. Brereton HM, Harland ML, Auld AM, Barritt GJ. Evidence that the TRP-1 protein is unlikely to account for store-operated Ca2+ inflow in Xenopus laevis oocytes. Mol Cell Biochem. 2000; 214:63-74. [PubMed: 11195791] 
33. Hedgecock EM, Culotti JG, Hall DH. The unc-5, unc-6, and unc-40 genes guide circumferential migrations of pioneer axons and mesodermal cells on the epidermis in C. elegans. Neuron. 1990; 4:61-85. [PubMed: 2310575]

34. Harris R, Sabatelli LM, Seeger MA. Guidance cues at the Drosophila CNS midline: identification and characterization of two Drosophila Netrin/UNC-6 homologs. Neuron. 1996; 17:217-228. [PubMed: 8780646]

35. Serafini $\mathrm{T}$, et al. The netrins define a family of axon outgrowth-promoting proteins homologous to C. elegans UNC-6. Cell. 1994; 78:409-424. [PubMed: 8062384]

36. Mitchell KJ, et al. Genetic analysis of Netrin genes in Drosophila: Netrins guide CNS commissural axons and peripheral motor axons. Neuron. 1996; 17:203-215. [PubMed: 8780645]

37. Serafini $\mathrm{T}$, et al. Netrin-1 is required for commissural axon guidance in the developing vertebrate nervous system. Cell. 1996; 87:1001-1014. [PubMed: 8978605]

38. Kennedy TE, Serafini T, de la Torre JR, Tessier-Lavigne M. Netrins are diffusible chemotropic factors for commissural axons in the embryonic spinal cord. Cell. 1994; 78:425-435. [PubMed: 8062385]

39. Li Y, et al. Essential role of TRPC channels in the guidance of nerve growth cones by brainderived neurotrophic factor. Nature. 2005; 434:894-898. [PubMed: 15758952]

40. Gordon XW, Poo M. Requirement of TRPC channels in netrin-1-induced chemo-tropic turning of nerve growth cones. Nature. 2005; 434:898-904. [PubMed: 15758951]

41. Clapham DE. TRP channels as cellular sensors. Nature. 2003; 426:517-524. [PubMed: 14654832]

42. Kolodziej PA, et al. frazzled encodes a Drosophila member of the DCC immunoglobulin subfamily and is required for CNS and motor axon guidance. Cell. 1996; 87:197-204. [PubMed: 8861904]

43. Keino-Masu K, et al. Deleted in colorectal cancer (DCC) encodes a netrin receptor. Cell. 1996; 87:175-185. [PubMed: 8861902]

44. Roberts A. Early functional organization of spinal neurons in developing lower vertebrates. Brain Res Bull. 2000; 53:585-593. [PubMed: 11165794]

45. Phelps PE, Alijani A, Tran TS. Ventrally located commissural neurons express the GABAergic phenotype in developing rat spinal cord. J Comp Neurol. 1999; 409:285-298. [PubMed: 10379921]

46. Roberts A, Dale N, Ottersen OP, Storm-Mathisen J. Development and characterization of commissural interneurones in the spinal cord of Xenopus laevis embryos revealed by antibodies to glycine. Development. 1988; 103:447-461. [PubMed: 3246217]

47. Ming G, Henley J, Tessier-Lavigne M, Song H, Poo M. Electrical activity modulates growth cone guidance by diffusible factors. Neuron. 2001; 29:441-452. [PubMed: 11239434]

48. Ming G, et al. Phospholipase C-gamma and phosphoinositide 3-kinase mediate cytoplasmic signaling in nerve growth cone guidance. Neuron. 1999; 23:139-148. [PubMed: 10402200]

49. Ren XR, et al. Focal adhesion kinase in netrin-1 signaling. Nat Neurosci. 2004; 7:1204-1212. [PubMed: 15494733]

50. Sayers LG, et al. Intracellular targeting and homotetramer formation of a truncated inositol 1,4,5trisphosphate receptor-green fluorescent protein chimera in Xenopus laevis oocytes: evidence for the involvement of the transmembrane spanning domain in endoplasmic reticulum targeting and homotetramer complex formation. Biochem J. 1997; 323:273-280. [PubMed: 9173893] 

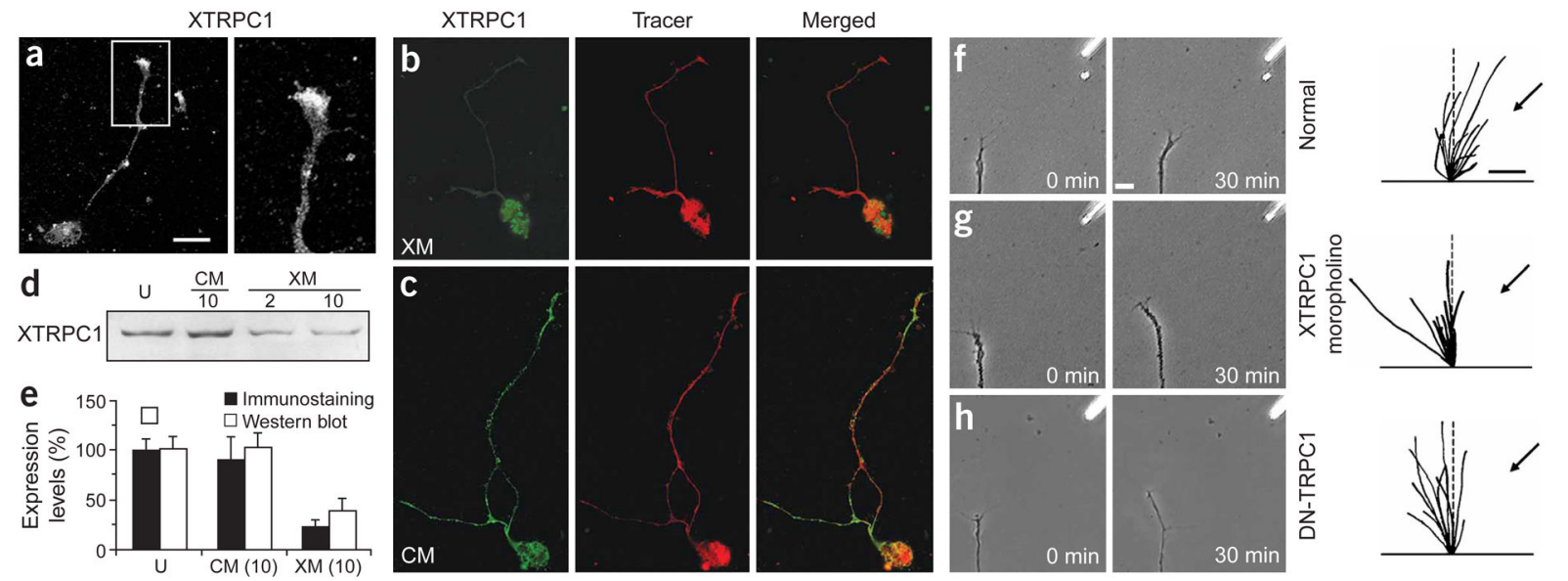

CM

Figure 1.

Requirement of XTRPC1 for growth cone turning responses to a gradient of netrin-1. (a) Sample confocal images of XTRPC1 staining in a cultured Xenopus spinal neuron (left) and an enlarged view of its growth cone (right). Scale bar: $40 \mu \mathrm{m}$ (left); 15 $\mu \mathrm{m}$ (right). (b-e) Knockdown expression of XTRPC1 by a specific morpholino. Sample confocal images show XTRPC1 staining (green) and a tracer (red) in cultured Xenopus spinal neurons derived from embryos injected with the XTRPC1 morpholino (XM, b) and a control morpholino (CM, c). Scale bar: $40 \mu \mathrm{m}$. A sample western blot (d) represents endogenous XTRPC1 in uninjected embryos (U), embryos injected with CM (10 ng per embryo), or embryos injected with XM (2 ng and 10

ng per embryo) at the one-cell stage. Data in e are from western blot of whole-embryo membrane preparations and immunostaining of growth cones of cultured spinal neurons (mean \pm s.d.; $n=4$ separated experiments). (f-h) Growth cone turning responses (left, center) in a gradient of netrin-1 of Xenopus spinal neurons derived from normal embryos (f), embryos injected with the XTRPC1 morpholino (g) or embryos injected with mRNA encoding a dominant-negative (DN) mutant form of mammalian TRPC1 (h) at the start ( $0 \mathrm{~min})$ and the end $(30 \mathrm{~min})$ of exposure to a netrin-1 gradient (5 $\mu \mathrm{g} / \mathrm{ml}$ in the pipette). Superimposed trajectories of neurite extension during the 30-min period for a sample population of ten neurons are shown at right. Arrows indicate direction of gradient. Scale bar: $10 \mu \mathrm{m}$. 

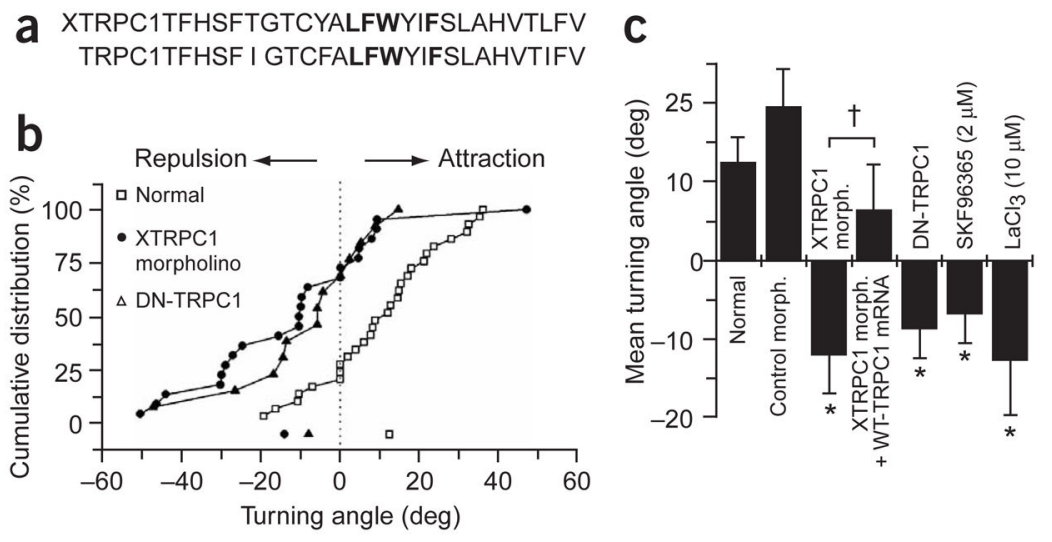

Figure 2.

Summary of growth cone turning responses to a gradient of netrin-1. (a) TRPCs are highly conserved in the proposed pore region of the channel. The amino acids in boldface are identical in XTRPC1 and mammalian TRPC1-TRPC7. (b) Growth cone turning in a gradient of netrin-1 in normal neurons or in neurons derived from embryos injected with the XTRPC1 morpholino or mRNA encoding DN-TRPC1. Shown are cumulative distributions of turning angles from individual experiments under various conditions. The mean turning angles are shown below. (c) Summary of growth cone turning angles under various conditions (mean \pm s.e.m.; $n=15-29$ ). * indicates statistically significant difference from normal neurons, and cross indicates significant difference between two conditions in bracket $(P<0.01$, bootstrap test). 
a

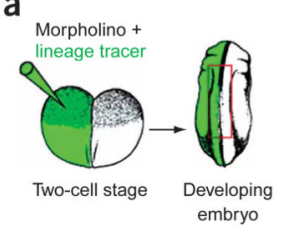

b

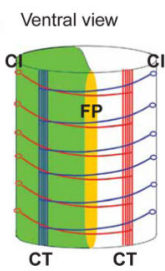

d

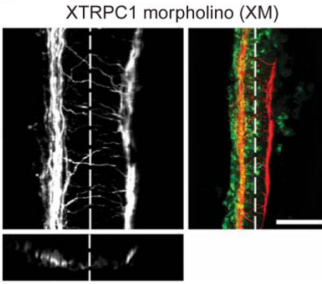

e

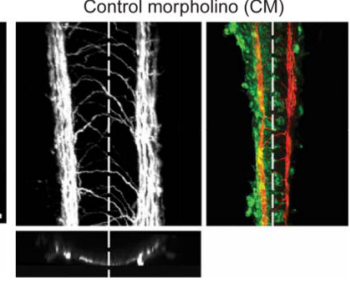

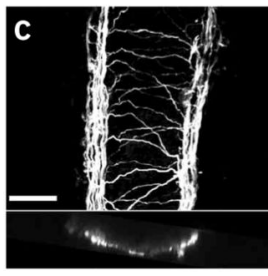

f

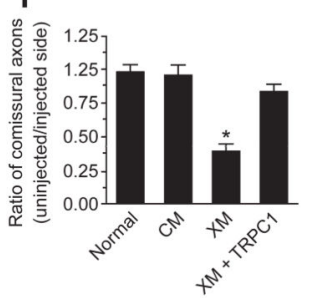

Figure 3.

XTRPC1 is required for formation of longitudinal axonal tracts by commissural interneurons in developing Xenopus spinal cord. (a) Microinjection at the two-cell stage allows specific manipulation of half of the embryo, with the uninjected half serving as the internal control. (b) Diagrams of commissural interneurons in developing Xenopus spinal cord (adapted from ref. 44). CI: commissural interneuron cell body; CT: commissural interneuron axon tracts; FP: floor plate. (c-e) Longitudinal axon tracts of commissural interneurons in developing Xenopus spinal cord from stage 28-30 embryos. Confocal $z$-stack projections with

3A10 staining of commissural interneuron axons from an uninjected embryo (c), embryos injected with the XTRPC1 morpholino (XM; d, left) or a control morpholino (CM; e, left), together with fixable FITC-dextran (green). Dashed lines indicate midline. Scale bar: $100 \mu \mathrm{m}$. Lower images: optical projections of the coronal section of ventral spinal cord. Images at right: larger portion of spinal cords with 3A10 staining of commissural axons (red) and the side of injection (green) at a smaller scale. Scale bar: $250 \mu \mathrm{m}$. (f) Comparison of commissural axons on uninjected and injected sides. Optical projections of coronal sections shown in $\mathbf{c}-\mathbf{e}$ were used for quantification (see Methods) for normal embryos or for embryos unilaterally injected with

$\mathrm{CM}, \mathrm{XM}$, or XM and mRNAs encoding mammalian TRPC1 at the two-cell stage (mean \pm s.e.m.; $n=6$ to 9 ). * indicates statistically significant difference from non-injected control $(P<0.01$, bootstrap test). 


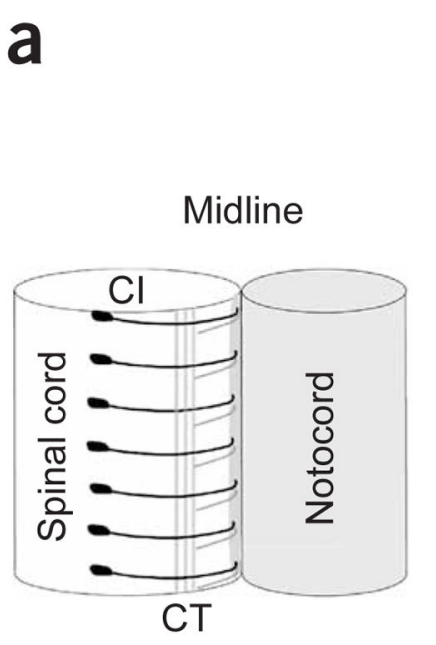

b

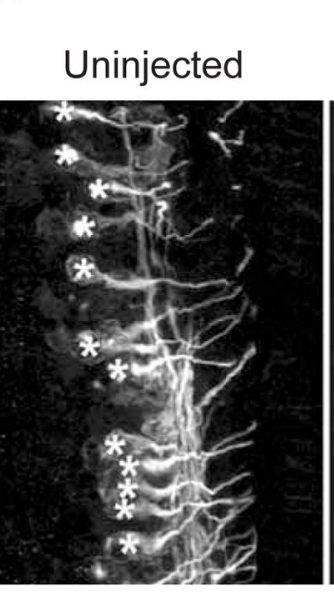

Control morpholino injected

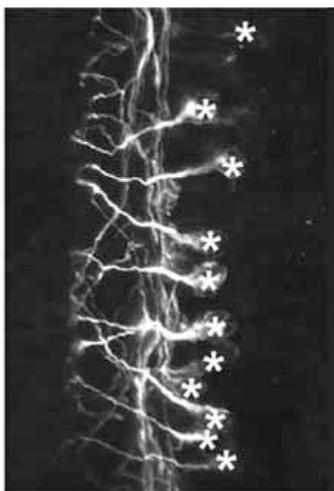

C XTRPC1 morpholino
injected

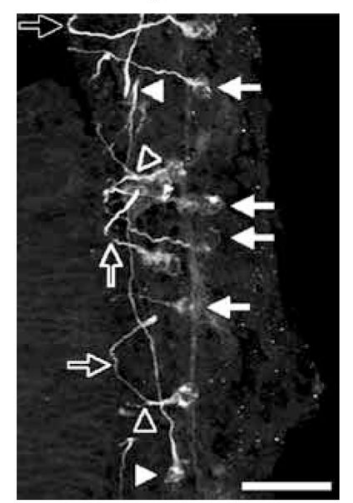

d
XTRPC1 morpholino injected
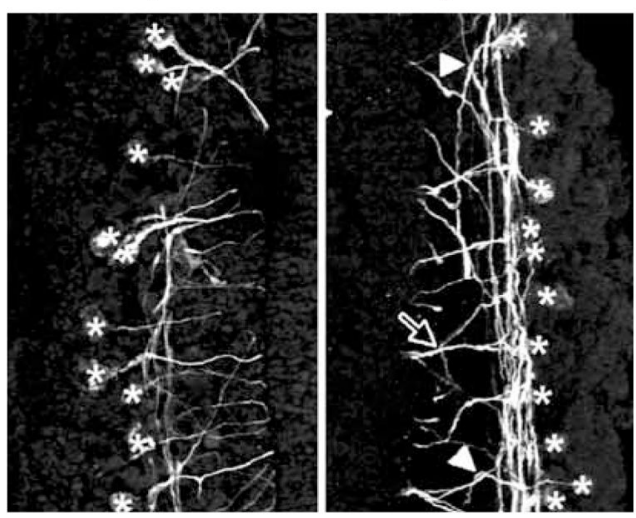

e

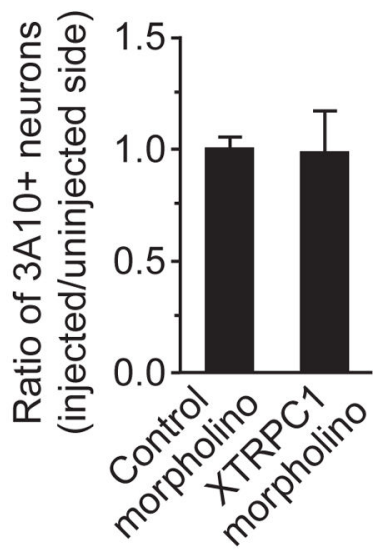

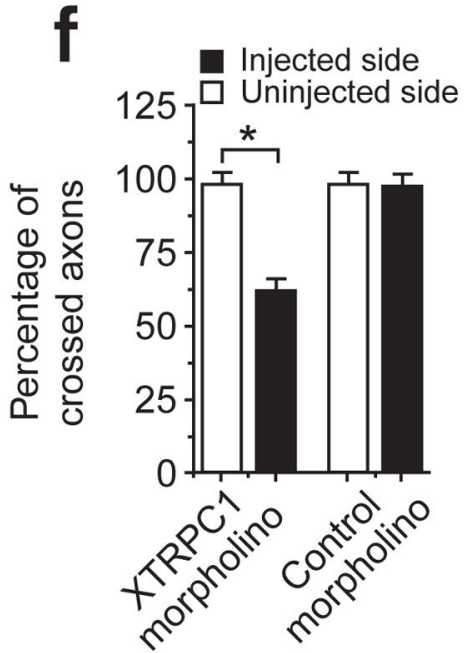

Figure 4.

XTRPC1 is required for attraction of commissural interneuron axons to the CNS midline. (a) Schematic sagittal view of commissural interneurons and their axons in the developing Xenopus spinal cord. CT: contralateral commissural interneuron axon. (b-d) Commissural interneuron projections in stage 23-25 embryos. Confocal $z$-stack projections of 3A10 staining of the uninjected and injected side within the same segment of the spinal cord, in embryos unilaterally injected with a control morpholino (b) or the XTRPC1 morpholino (d) at the two-cell stage. Scale bar: $40 \mu \mathrm{m}$. Cell bodies of commissural interneurons show 3A10 staining (*). Different phenotypes of trajectories of commissural interneurons based on three-dimensional reconstruction of $z$-stack confocal images: crossed midline (filled arrows); stalled or turned back from midline (open arrows); joined the ipsilateral (IL) tract (filled triangles); prematurely branched before crossing midline (open triangles). (e) Comparison of the density of 3A10-positive commissural interneurons derived from the injected and uninjected side within the same segment of the developing spinal cord (mean \pm s.d.; $n=9$ ). (f) Summary of phenotypes of midline axon guidance behavior of 3A10positive commissural interneurons in embryos unilaterally injected with the XTRPC1 morpholino or with control morpholino at the two-cell stage (mean \pm s.e.m.; $n=10-23)$. ${ }^{*}$ indicates statistically significant difference $(P<0.01$, bootstrap test). 

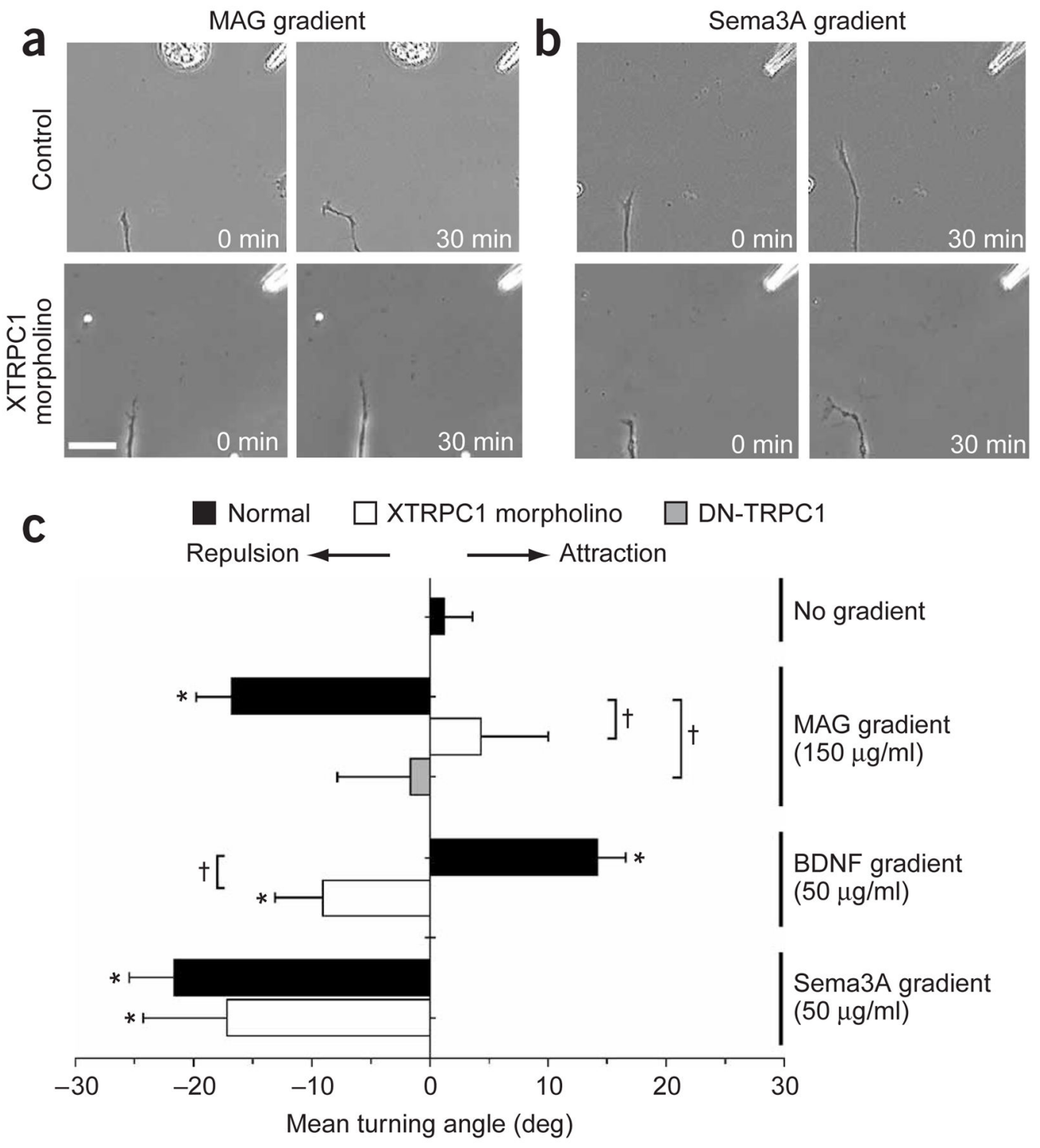

Figure 5.

XTRPC1 as a general mediator for $\mathrm{Ca}^{2+}$-dependent neuronal growth cone guidance. (a) Growth cone turning in a gradient of MAG. Shown are sample images of growth cones at the start $(0 \mathrm{~min})$ and the end $(30 \mathrm{~min})$ of exposure to a gradient of MAG $(150 \mu \mathrm{g} / \mathrm{ml}$ in the pipette) for a normal neuron (top) and a neuron derived from embryos injected with the XTRPC1 morpholino (bottom). Scale bar: $20 \mu \mathrm{m}$. (b) Growth cone turning responses in a gradient of Sema3A. Similar to a, except that a Sema3A gradient was used $(50 \mu \mathrm{g} / \mathrm{ml}$ in the pipette). (c) Summary of mean turning angles under different conditions (mean \pm s.e.m.; $n=$ 15-30). * indicates statistically significant difference from the control (no gradient control), and crosses indicate statistically significant difference between conditions in brackets $(P<0.01$, bootstrap test $)$. 